\title{
Methodology Report \\ Differential Proteome Analysis of the Preeclamptic Placenta Using Optimized Protein Extraction
}

\author{
Magnus Centlow, ${ }^{1}$ Stefan R. Hansson, ${ }^{1}$ and Charlotte Welinder ${ }^{2}$ \\ ${ }^{1}$ Department of Obstetrics \& Gynecology, Lund University, BMC C14, 22184 Lund, Sweden \\ ${ }^{2}$ Department of Oncology, Lund University, Barngatan 2B, 22185 Lund, Sweden
}

Correspondence should be addressed to Charlotte Welinder, charlotte.welinder@med.lu.se

Received 17 May 2009; Accepted 27 June 2009

Academic Editor: Benjamin A. Garcia

Copyright (C) 2010 Magnus Centlow et al. This is an open access article distributed under the Creative Commons Attribution License, which permits unrestricted use, distribution, and reproduction in any medium, provided the original work is properly cited.

\begin{abstract}
The human placenta is a difficult tissue to work with using proteomic technology since it contains large amounts of lipids and glycogen. Both lipids and glycogen are known to interfere with the first step in the two-dimensional polyacrylamide gel electrophoresis (2D-PAGE), the isoelectric focusing. In order to gain the best possible protein separation on 2D-PAGE, an optimized sample preparation protocol for placental proteins was developed. Two different buffers, urea/CHAPS and Hepes, were used for solubilization in combination with six different precipitation methods. The removal of glycogen from the samples by centrifugation was crucial for the final proteome maps. Solubilization with urea/CHAPS in combination with dichloromethane/methanol or acidified acetone proved to be the best precipitation procedures. When applied to clinical placenta samples apolipoprotein A1 was found to be accumulated in the preeclamptic placenta, where it may either have a nutritional effect or act as a modifier of signal transduction.
\end{abstract}

\section{Introduction}

The human placenta is unique among species. As a matter of fact, of all mammalian organs the placenta shows the greatest variation in terms of anatomy. However, in all species it has the same fundamental function, to nourish the growing fetus, by establishing contact with the maternal blood circulation [1]. In addition, the placenta acts as a protective barrier; as an example it prevents stress hormones to pass over to the fetus via active transport [2]. In order to prevent rejection of the fetus, the placenta expresses an intricate pattern of major histocompatibility complex molecules, immunizing the mother against the foreign fetal tissue [3]. During pregnancy the placenta also has several important endocrine functions, producing important hormones such as human chorionic gonadotropin and human placental lactogen $[4,5]$. Hence, due to its vast number of functions the placenta expresses more than 20000 DNA sequences and is perhaps the organ expressing the largest number of genes [6]. In fact, it has been used as a "fishing pond" for finding and sequencing new genes and has been used to identify and clone transporters and receptors, such as human growth hormone-variants, integrin alpha $\mathrm{V}$, and subtypes of the angiotensin receptors [7-9].

Preeclampsia (PE) is a pregnancy associated disorder characterized by hypertension and proteinuria. It affects 3\%$7 \%$ of all pregnancies [10] and is a major cause of maternal and fetal mortality. Treatment for PE is symptomatic, and removal of the placenta is the only curative treatment, suggesting that the placenta is a key in the development of $\mathrm{PE}$. The etiology of PE is still largely unknown; however, it is generally accepted that PE begins with inadequate placentation. Invasion of placental trophoblast cells into the maternal spiral arteries is too shallow, leading to inadequate placental perfusion and hypoxia $[11,12]$.

Proteomics is a research field undergoing rapid development, especially in regards to the methods being used. The most common method is two-dimensional polyacrylamide gel electhrophoresis (2D-PAGE). 2D-PAGE for proteome studies gives a map of proteins which reflect changes in 
protein expression levels, as well different isoforms and posttranslational modifications. Lately, three types of protein microarrays have become available, namely, analytical microarrays, functional microarrays, and reverse phase microarrays (reviewed in [13]). Highly sensitive and specific recombinant antibody microarrays allowing screening of crude protein extracts are also being developed [14]. Due to the large number of proteins, no protein array platform has yet been designed that covers the entire human placental proteome. Thus, when screening the placental proteome 2D-PAGE is still among the most accepted methods giving the highest coverage although this may soon change. However, there are several obstacles when working with placental tissue, since it contains large amounts of lipids and glycogen [15]. In order for the first dimension, isoelectric focusing (IEF), to be successful, proteins have to be efficiently extracted, and solubilized, and contaminants, such as lipids, polysaccharides, nucleic acids, and salt ions, must be removed. Lipids, polysaccharides and nucleic acids bind to proteins which increases the viscosity of the samples and often results in horizontal streaking on the 2D-PAGE. If the salt concentration is too high in the sample, the conductivity in the IPG strips increases and prolongs the IEF, resulting in poor 2D-PAGE resolution. These contaminants can be removed by precipitation of the solubilized samples. Unfortunately, there is no universal precipitation method that removes all the different contaminants. For example, precipitating samples with dichloromethane/methanol has been described to remove lipids whereas precipitation with ethanol will remove nucleic acids [16]. For these reasons, the placenta proteome is not fully investigated although some progress has been made in the use of proteomics technology to study PE placenta $[17,18]$.

The primary aim of this study was to establish a robust sample preparation protocol of human placental tissue, by comparing two different solubilization solutions in combination with six different precipitation methods.

\section{Material and Methods}

2.1. Tissue Samples. Sixty women admitted at the Department of Obstetrics and Gynecology, Lund University Hospital, were included and assigned to two groups: PE ( $n=$ $30)$ and control $(n=30)$ (Table 1). All women gave their written informed consent. PE was defined as blood pressure above $140 / 90 \mathrm{mmHg}$ and proteinuria above $0.3 \mathrm{~g} / \mathrm{L}$ or rise in blood pressure above $20 \mathrm{mmHg}$ from the first trimester of pregnancy [19]. No women in the control group suffered $\mathrm{PE}$ in a previous pregnancy. Patients were matched based on gestational age. A $10 \mathrm{~mm}$ cube of placenta tissue was collected immediately after removal of the placenta and was frozen on dry ice and stored at $-80^{\circ} \mathrm{C}$. Patients with other systemic diseases were excluded from the study. This study was approved by the Ethical Committee Review Board for studies in human subjects at Lund University.

2.2. Protein Extraction. Human placental tissues were pulverized, and the frozen placental powder ( $25 \mathrm{mg}$ frozen weight) was homogenized with a lysis solution containing either $3 \mathrm{~mL}$
TABLE 1: Clinical characteristics of the patients participating in this study. Age and Gestational length are expressed as median (range), and the other values as mean \pm standard deviation.

\begin{tabular}{lcc}
\hline & Controls & PE \\
\hline$n$ & 30 & 30 \\
Age (years) & $32(23-44)$ & $30(22-45)$ \\
Gestational length (days) & $269(247-295)$ & $265(235-287)$ \\
Systolic MAP (mmHg) & $116 \pm 11.3$ & $150 \pm 12.1$ \\
Diastolic MAP (mmHg) & $67 \pm 4.4$ & $103 \pm 7.9$ \\
Albumin (g/l) & $\mathrm{ND}$ & $1.9 \pm 1.4$ \\
Placenta weight (g) & $687 \pm 144$ & $631 \pm 128$ \\
Child gender (M : F) & $19: 11$ & $15: 15$ \\
Child weight $(\mathrm{g})$ & $3671 \pm 297$ & $3219 \pm 571^{\mathrm{a}}$ \\
\hline
\end{tabular}

ND: not detected, $\mathrm{M}:$ male, $\mathrm{F}$ : female.

${ }^{\mathrm{a}}$ Student two-tailed unpaired $t$-test showed a significant difference between the control and the PE group: $P<.0002$.

$8 \mathrm{M}$ urea and 2\% CHAPS or $3 \mathrm{~mL}$ ice cold $10 \mathrm{mM}$ Hepes, $\mathrm{pH}$ 7.5, $24 \mathrm{mM} \mathrm{KCl}$ supplemented with a protease inhibitor cocktail (Complete mini plus EDTA, Roche, Basel, Switzerland) [20]. Samples were stirred for 1 hour followed by centrifugation at $43000 \mathrm{~g}$ for 2 hours. Protein concentrations were determined by the bicinchoninic acid (BCA) method (Thermo Scientific, Rockford, USA) with bovine serum albumin (BSA) as a standard. Pooled samples were prepared by combining equal amounts of proteins from individual samples. The pooled samples were either used directly (fresh samples) or stored at $-80^{\circ} \mathrm{C}$ prior to use (frozen samples). The individual samples were stored at $-80^{\circ} \mathrm{C}$.

2.3. Protein Precipitation Procedures. Proteins were precipitated according to the following procedures. For 1D-PAGE, $30 \mu \mathrm{g}$ were precipitated, and for small and large 2D-PAGE, $100 \mu \mathrm{g}$ and $500 \mu \mathrm{g}$ were precipitated, respectively.

Acetone. Extracted samples were mixed with ice-cold acetone to final concentration of $80 \%$ acetone. Samples were incubated overnight at $-20^{\circ} \mathrm{C}$ followed by centrifugation at $9000 \mathrm{~g}$ for 2 minutes. The supernatant was removed, and the protein pellets were allowed to air dry.

Acidified Acetone. Samples were precipitated as above, but the acetone was acidified with acetic acid to a final concentration of $0.05 \%$.

Ethanol. Ten volumes of ice-cold ethanol were added to one volume of sample. Samples were centrifuged as above, and the protein pellets were allowed to air-dry.

Dichloromethane/Methanol. Samples were precipitated as previously described [16]. Briefly, one volume of sample was mixed with 4 volumes of methanol, 2 volumes of dichloromethane, and 3 volumes of distilled water. The samples were mixed between each addition. The samples were centrifuged $9000 \mathrm{~g}$ for 2 minutes at room temperature (RT). The supernatant was discarded, and 3 volumes of methanol were added followed by mixing. The samples were 
centrifuged at $9000 \mathrm{~g}$ for 2 minutes and the supernatant was again discarded after which pellets were air dried.

Trichloroacetic Acid (TCA). One volume of 40\% TCA was added to one volume of samples and incubated for 1 hour on ice followed by centrifugation at $9000 \mathrm{~g}$ for 2 minutes. The supernatant was removed, and the protein pellets were either directly dried or washed with ethanol and then allowed to air dry. The dried pellets were either dissolved in $30 \mu \mathrm{L}$ sodium dodecyl sulphate (SDS) sample buffer pH 8.5 (247 mM Tris, 2\% SDS, $0.5 \mathrm{mM}$ edetate (EDTA), $16.5 \mathrm{mM}$ dithiothreitol (DTT), 10\% glycerol, and $0.025 \%(\mathrm{w} / \mathrm{v})$ bromophenol blue) followed by 1D-PAGE or dissolved in rehydration sample buffer containing $8 \mathrm{M}$ Urea, 2\% CHAPS, $10 \mathrm{mM}$ DTT, and 2\% 3-10 NL ampholines (GE Healthcare, Little Chalfont, UK).

2.4. 1D-PAGE. Samples $(30 \mu \mathrm{g})$ were loaded onto 10-well $4 \%-12 \%$ NuPAGE Bis-Tris gels (Invitrogen, Carlsbad, USA) and run at $200 \mathrm{~V}$ for 45 minutes using 2-( $N$-morpholino) ethanesulfonic acid (MES) as running buffer. Proteins were visualized by staining with Coomassie Brilliant Blue (R-350).

2.5. 2D-PAGE. The first dimensional isoelectric focusing (IEF) was performed on a horizontal Multiphor (Amersham Biosciences, USA). Immobilized $\mathrm{pH}$ gradient (IPG) Blue native strips $3-10 \mathrm{NL}(0.5 \times 3 \times 70 \mathrm{~mm}$ or $0.5 \times 3 \times 240 \mathrm{~mm})$ (SERVA Electrophoresis GmbH, Heidelberg, Germany) were in-gel rehydrated in rehydration sample buffer over night. For $7 \mathrm{~cm}$ strips, $130 \mu \mathrm{L}$ (containing $100 \mu \mathrm{g}$ of proteins) and for $24 \mathrm{~cm}$ strips, $450 \mu \mathrm{L}$ (containing $500 \mu \mathrm{g}$ of proteins) rehydration sample buffer were used. The focusing was performed at the following voltage gradients: $150 \mathrm{~V}$ for 30 minutes, $300 \mathrm{~V}$ for 30 minutes, $600 \mathrm{~V}$ for 30 minutes, $1500 \mathrm{~V}$ for 1.5 hours, $3000 \mathrm{~V}$ for 2 hours $(7 \mathrm{~cm}$ strips) and 25 hours ( $24 \mathrm{~cm}$ strips). The strips were equilibrated for 10 minutes in a solution containing $65 \mathrm{mM}$ DTT, $6 \mathrm{M}$ urea, $30 \%(w / v)$ glycerol, $2 \%(w / v)$ SDS and $50 \mathrm{mM}$ Tris- $\mathrm{HCl}$ $\mathrm{pH}$ 8.8. A second equilibration step was performed for 10 minutes in the same solution except for DTT, which was replaced by $259 \mathrm{mM}$ iodoacetamide. The strips were soaked in electrophoresis buffer $(24 \mathrm{mM}$ Tris base, $0.2 \mathrm{M}$ Glycine and $0.1 \%$ SDS) just before the second dimensional gel electrophoresis. The strips were applied on $12 \%$ homogeneous Tris-glycine gels $(0.1 \times 8 \times 8 \mathrm{~cm}$ or $0.1 \times 20 \times 24 \mathrm{~cm})$ and overlaid with a solution of $1 \%$ agarose in electrophoresis buffer (kept at $60^{\circ} \mathrm{C}$ ). Electrophoresis was carried out in an XCell Mini-cell apparatus (Invitrogen) using electrophoresis buffer at $125 \mathrm{~V}$ for 1.5 hours or in an Hoefer DALT gel apparatus (GE Healthcare) at $20^{\circ} \mathrm{C}$ and constant $80 \mathrm{~V}$ for 19 hours.

Gel Staining. Gels were either stained with silver [21] or stained with SYPRO Ruby (Thermo Scientific).

Image Analysis. Small gels were scanned using a CanoScan 995OF (Canon, Solna, Sweden), and large gels were scanned using an Amersham Typhoon 9400 (GE Healthcare). The images of the large gels stained with SYPRO Ruby were submitted to Ludesi 2D Analysis (Ludesi, Malmo, Sweden, $\mathrm{http://www.ludesi.com)} \mathrm{for} \mathrm{spot} \mathrm{detection,} \mathrm{matching,} \mathrm{and}$ analysis.

Peptide Mass Fingerprinting. The spots of interest were washed and digested as previously described [22]. The purified peptides were eluted directly onto the sample target (Anchorchip target, Bruker Daltonik GmbH, Bremen, Germany) where $0.7 \mu \mathrm{L}$ of matrix, 2,5-dihydroxybenzoic acid $(10 \mathrm{mg} / \mathrm{ml}$ in $30 \% \mathrm{ACN})$ had been added. Mass spectra of positively charged ions were recorded on a Bruker Reflex III instrument (Bruker Daltonik) operated in the reflector mode. A total of 160-210 single shot spectra were accumulated from each sample. The XMASS 5.0 and MS Biotools software packages provided by the manufactures were used for data processing. Known autoproteolysis products from the trypsin were used for internal calibration.

Database Searching. For protein identification, human protein sequences in the SwissProt database were searched using the Mascot Software (Matrix Science Ltd., London, UK). Parameters specified in the search were taxa, Homo Sapiens; missed cleavages, 1 ; peptide mass tolerance $+/ 0.1 \mathrm{Da}$; fixed modification, carbamidomethyl (C); variable modification, oxidized methionine.

2.6. Western Blot. Proteins were separated by $1 D-P A G E$ using 10-well 12\% NuPAGE Bis-Tris gels (Invitrogen) according to manufacturer's instruction. The gels were loaded with $2.5 \mu \mathrm{g}$ protein per well and run for 60 minutes at $200 \mathrm{~V}$ in MOPS running buffer under reduced conditions and blotted onto polyvinylidene fluoride (PVDF) membranes for 8 minutes at $23 \mathrm{~V}$ using an iBlot dry blotting system (Invitrogen). Membranes were incubated with primary antibody for 1 hour (mouse monoclonal anti-APOA1, sc-69755, $1: 2000$ dilution), followed by washes and then, incubated with the secondary antibody for 1 hour (goat polyclonal antimouse IgG, sc-2031, $1: 5000$ dilution). Antibodies were obtained from Santa Cruz (Santa Cruz Biotechnology, Santa Cruz, USA). Subsequently, the membrane was exposed to Enhanced chemilumeniscence ECL+ (GE Healthcare) for 3 minutes. Autoradiographic film Hyperfilm ECL (GE Healthcare) was applied to the blot for various durations to obtain satisfactory exposure. When developed, films were scanned with ultra violet light using a Gene Flash scanner (Syngene, Cambridge, UK) and imported into Syngene Gene Tools. One reference sample was added to all gels to serve as an internal calibrator to normalize the quantification between gels. A students $t$-test was used to determine statistical difference between the two groups. A $P$-value $<.05$ was considered statistically significant.

2.7. Immunohistochemistry. Immunohistochemistry was performed using EnVision+System-HRP (DakoCytomation, Carpinteria, USA) on 4 representative PE samples and 4 controls according to the manufacturer's instructions. Briefly, fresh frozen sections, $12 \mu \mathrm{m}$ thick, of the placenta 


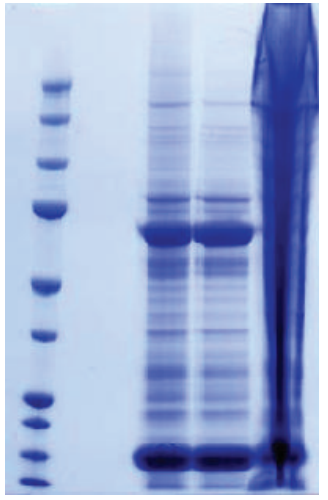

(a)

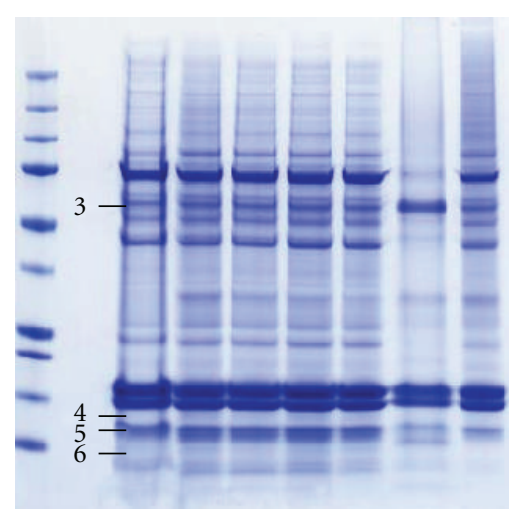

(b)

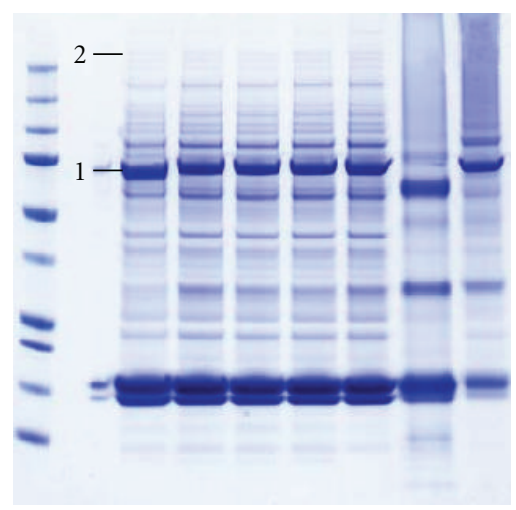

(c)

FIGURE 1: 1D-PAGE comparing solubilization and precipitation methods. (a) One-dimensional separation of proteins in PE placenta solubilized in Hepes buffer before and after centrifugation at $43000 \mathrm{~g}$. From left to right: Mw Precision standard marker, starting material $(30 \mu \mathrm{g})$, supernatant after centrifugation $(30 \mu \mathrm{g})$, and pellet after centrifugation. The gel was stained with Coomassie Brilliant blue. (b) Onedimensional separation of proteins in PE placenta solubilized in urea/CHAPS solution and (c) Hepes buffer. The lanes are from left to right: MW precision standard markers, starting material, and precipitations with acetone, acidified acetone, ethanol, dichloromethane/methanol, TCA, and TCA followed by ethanol wash. Bands indicated with numbers were identified by MALDI-TOF MS (Table 2).

TABle 2: Proteins identified using MALDI-TOF MS.

\begin{tabular}{|c|c|c|c|c|c|c|}
\hline & Protein & Acc. No & $\begin{array}{c}\mathrm{Mw} \\
(\mathrm{kDa})\end{array}$ & $\begin{array}{c}\text { Matched } \\
\text { peaks }\end{array}$ & Score & $\begin{array}{c}\text { Seq. Cov } \\
(\%)\end{array}$ \\
\hline \multirow{3}{*}{ Band 1} & Annexin A1 & P04083 & 38.9 & 18 & 117 & 59 \\
\hline & Annexin A2 & P07355 & 38.8 & 20 & 92 & 45 \\
\hline & Glyceraldehyd-3-phosphate dehydrogenase & P04406 & 36.2 & 13 & 59 & 41 \\
\hline \multirow[t]{2}{*}{ Band 2} & Endoplasmin & P14625 & 92.7 & 15 & 99 & 19 \\
\hline & Keratin, type I cytoskeletal 18 & P05783 & 48.0 & 22 & 142 & 37 \\
\hline \multirow[t]{2}{*}{ Band 3} & Keratin, type II cytoskeletal 8 & P05787 & 53.7 & 15 & 77 & 26 \\
\hline & Vimentin & P08670 & 53.7 & 28 & 207 & 52 \\
\hline \multirow{3}{*}{ Band 4} & Hemoglobin subunit gamma-1 & P69891 & 16.2 & 8 & 86 & 42 \\
\hline & Hemoglobin subunit gamma-2 & P69892 & 16.2 & 9 & 81 & 51 \\
\hline & Hemoglobin subunit alpha & P69905 & 15.3 & 6 & 66 & 53 \\
\hline \multirow[t]{2}{*}{ Band 5} & Hemoglobin subunit gamma-1 & P69891 & 16.2 & 8 & 78 & 46 \\
\hline & Hemoglobin subunit gamma-2 & P69892 & 16.2 & 8 & 78 & 46 \\
\hline Band 6 & Hemoglobin subunit alpha & P69905 & 15.3 & 8 & 72 & 59 \\
\hline Spot PE & Apolipoprotein A1 & P02647 & 30.7 & 7 & 76 & 26 \\
\hline Spot Ctl & Tropomyosin alpha- 3 chain & P06753 & 32.9 & 9 & 63 & 26 \\
\hline
\end{tabular}

samples were fixed by immersion in $4 \%$ phosphate buffered saline (PBS) buffered formaldehyde for 15 minutes at RT. Sections were incubated in a blocking solution containing $3 \% \mathrm{H}_{2} \mathrm{O}_{2}$ for 10 minutes at RT. The APOA1 antibody was diluted $(0.1 \mu \mathrm{g} / \mathrm{mL})$ in PBS containing $0.25 \%$ Triton X100 and $1.5 \%$ normal goat serum. Sections were incubated over night at $4^{\circ} \mathrm{C}$ after which the sections were rinsed. Peroxidase labeled polymer was applied to cover the specimens and then incubated for 30 minutes. After rinsing the sections, LiquidDAB substrate chromogen solution was applied and incubated for 8 minutes. The sections were rinsed and stained with Mayers hematoxylin after which sections were mounted with Mountex (Histolab, Gothenburg, Sweden) and cover slipped. Sections were viewed under an Olympus BX-60 microscope and images captured using an Olympus
DP50CU digital camera (Olympus America Inc., Center Valley, USA).

\section{Results}

3.1. Solubilization and Precipitation. Two solubilization solutions were chosen: Hepes buffer [20] and the commonly used urea/CHAPS. A centrifugation step was added to remove contaminants such as glycogen and cell debris. The pellet resulted in smearing on 1D-PAGE (Figure 1(a)) and without the centrifugation step; no proteins entered the IEF-strip resulting in a blank 2D-PAGE (data not shown). There were no obvious differences in protein patterns between the supernatant and the starting material (Figure 1(a)). 1DPAGE separation was used to determine protein expression 

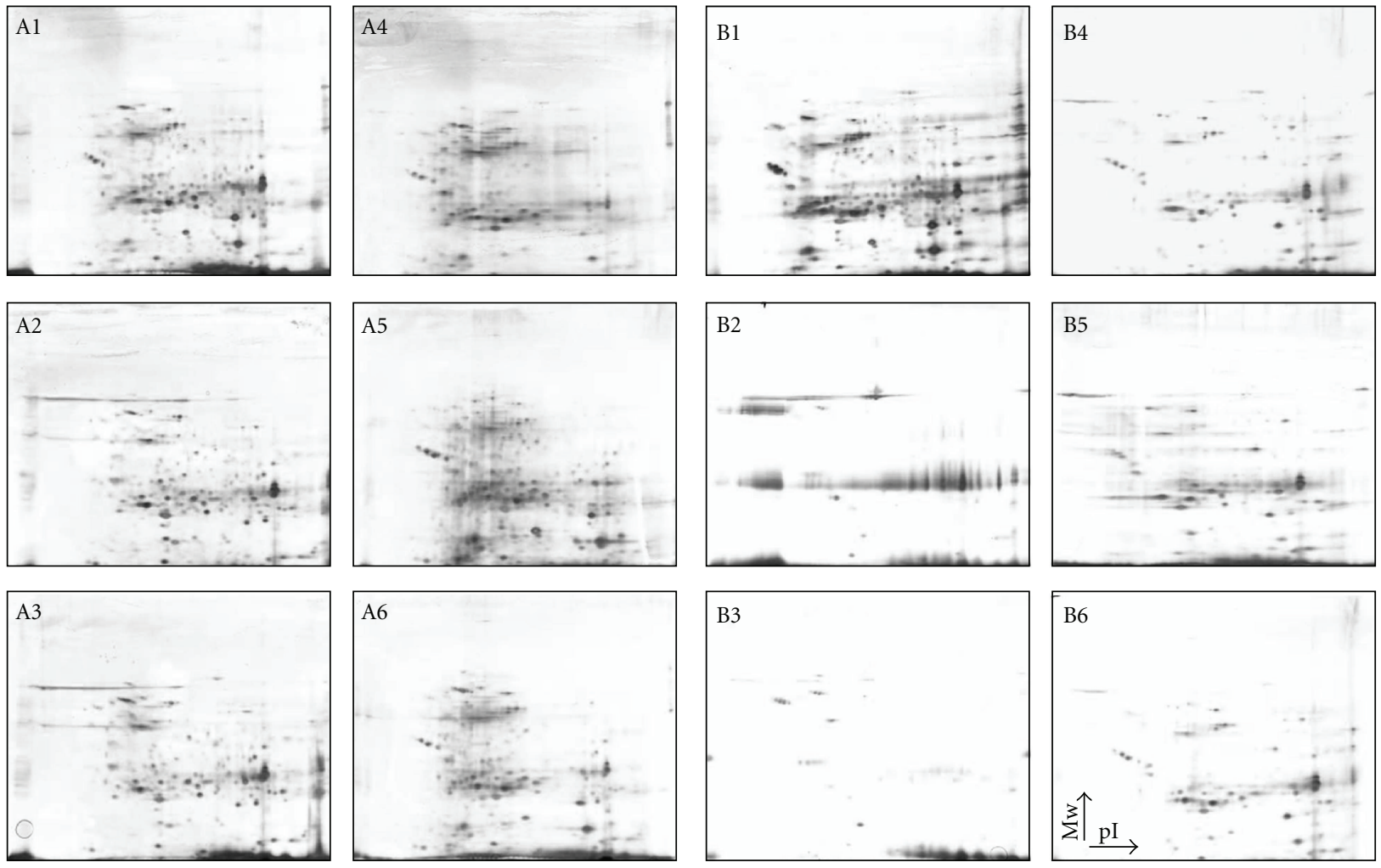

(a)

(b)

FIgure 2: Two-dimensional separation of precipitated proteins ( $100 \mu \mathrm{g})$ in PE placenta solubilized in (a) urea/CHAPS and (b) Hepes buffer. Precipitation procedures (1) dichloromethane/methanol, (2) TCA, (3) TCA followed by ethanol wash, (4) acetone, (5) acidified acetone, and (6) ethanol. The gels were stained with silver. Arrows on lower right image represent directions for increasing Mw and pI. Directions are the same for all gels.

from the two different solubilization solutions. A major difference in the protein pattern between Hepes buffer and urea/CHAPS was obtained in the low molecular mass range. Minor differences at higher molecular mass range are also obtained when comparing the two different solubilization solutions (Figures 1(b) and 1(c)). A number of the protein bands that visibly differed between the two solubilization solutions were identified using MALDI-TOF MS. The obtained identities included annexin A1, annexin A2, endoplasmin, vimentin, and hemoglobin subunit alpha and gamma (Table 2). Some of the bands from the 1D-PAGE contained more than one identity due to the limited power of resolution using 1D-PAGE.

Six different precipitation procedures were tested: acetone, acidified acetone, ethanol dichloromethane/methanol, TCA, and TCA followed by ethanol wash. To determine the effects of precipitation procedures on protein patterns, precipitated samples were analyzed on 1D-PAGE gels (Figures 1(b) and 1(c)). Samples precipitated with TCA, with or without ethanol wash, showed a different protein expression profile compared to the other four precipitation methods. No significant differences in the 1D-PAGE expression profiles were detected between the other four precipitation procedures (acetone, acidified acetone, ethanol and dichloromethane/methanol).
3.2. 2D-PAGE. Samples solubilized with urea/CHAPS generally resulted in more protein spots and less horizontal streaking, regardless of precipitation procedure (Figure 2). The investigation of whether freezing of solubilized and centrifuged samples influenced the results, both fresh and frozen samples, was analyzed using 2D-PAGE. No major differences could be detected in the protein expression profiles for any of the different solubilization or precipitation methods (data not shown). An increased number of low molecular weight proteins were obtained with samples solubilized in urea/CHAPS precipitated with acidified acetone (Figure 2, A5).

These spots were not obtained with other combinations of solubilizations and precipitations (Figure 3). Electroendosmotic flow (EOF) was detected during the IEF run with samples precipitated with acetone, acidified acetone, or ethanol. This resulted in swelling of the IPG-strip in the alkaline $\mathrm{pH}$ gradient and might cause a poor protein separation at an isoelectric point above 8 .

3.3. Preeclampsia. Urea/CHAPS in combination with either acidified acetone or dichloromethane/methanol precipitation was judged to be the most efficient extraction methods for 2D-PAGE. However, we chose dicholoromethane/methanol since it is known to remove lipids of which 


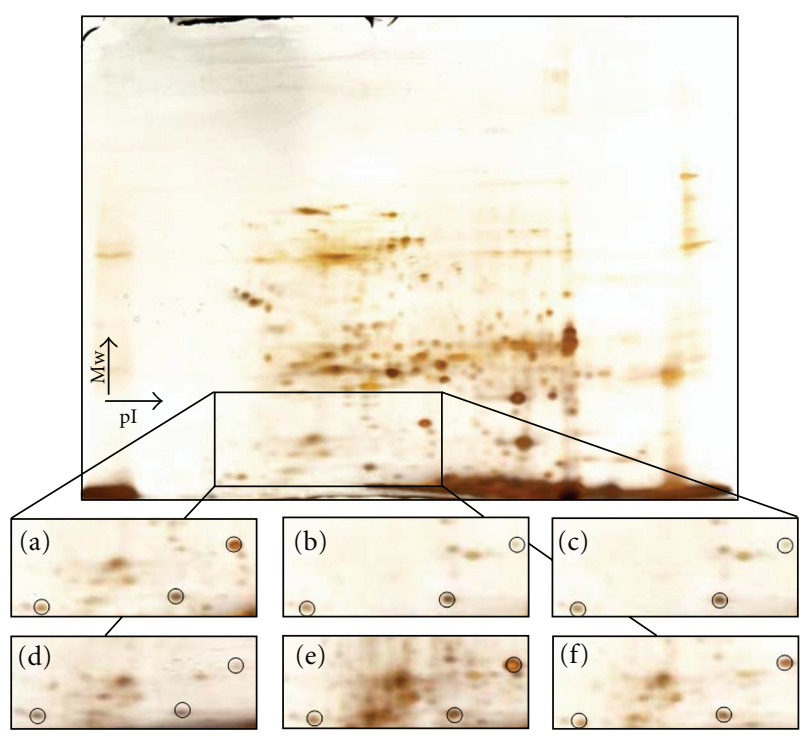

FIgURE 3: Two-dimensional separation of precipitated proteins $(100 \mu \mathrm{g})$ in PE placenta solubilized in urea/CHAPS. Segments show differential expression pattern in consequence of precipitation procedures. (a) dichloromethane/methanol, (b) TCA, (c) TCA followed by ethanol wash, (d) acetone, (e) acidified acetone, and (f) ethanol. Circles indicate landmarks for orientation. The gels were stained with silver.

there is abundance in the placenta. In a first attempt to investigate whether it is possible to detect differences between the PE and normal placenta in crude extracts, we pooled crude placenta extracts from 30 preeclamptic and 30 normotensive women respectively. In order to decrease the variation of the differently expressed proteins on the $2 \mathrm{D}$ PAGE, three replicates of each pooled group were made. The number of spots detected was 604 and 747 for preeclamptic and normotensive, respectively. The number of spots was calculated based on the criteria that the spots were present in all triplicate gels in each group. The analysis of the 2DPAGE showed that 23 proteins were increased and 28 were decreased in the PE placenta (Figure 4). Two proteins have so far been identified with MALDI-TOF MS: apolipoprotein A1 (APOA1) (acc number: P02647) and tropomyosin-3 (TPM3) (acc number: P06753). APOA1 was 1.63 times increased in the PE placenta pool as compared to the control pool (PE: $10871 \pm 1340$ optical density (OD) units and controls: $6663 \pm$ 1772 OD units values presented as mean \pm SD). TPM3 was only found in the normotensive pool.

The increased accumulation of APOA1 in the PE placenta was validated by means of western blot (Figure 5(a)). All samples, $30 \mathrm{PE}$ and 30 controls, were run individually. After measuring the absorption of all the APOA1 bands, APOA1 was found to be 1.18 times increased in PE (PE: $63120 \pm$ 14350 optical density (OD) units and controls: $52810 \pm$ 13310 OD units values presented as mean \pm SD). The accumulation of APOA1 was significantly increased in PE $(P=$ 0.02) (Figure 5(b)). Immunohistochemistry showed APOA1 to be accumulated in trophoblasts. Both normotensive and
PE placenta sections showed a similar pattern with weaker staining in the control sections (Figures $5(\mathrm{c})-5(\mathrm{e})$ ). The TPM3 expression could not be validated by means of Western blot since the recommended antibodies did not work in our hands (sc-32516 and sc-18174, Santa Cruz Biotechnology, Santa Cruz, USA).

\section{Discussion}

Due to the vast number of functions and many different cell types besides the trophoblasts, including white and red blood cells and endothelial cells, the placenta is a complex organ to study. In order to optimize the protein sample preparation for 2D-PAGE, we compared two different solubilization solutions in combination with six different precipitation methods in order to obtain maximal separation of the proteins.

Hepes buffer and urea/CHAPS showed different protein patterns in the low molecular mass range. Although no protease inhibitors were added to the urea/CHAPS solution, urea denatures and inactivates proteases and thus, it is unlikely that the differences are due proteolytic cleavage [23]. Several steps in the sample preparations appear to be important to remove contaminating substances. During pregnancy there is a gradual accumulation of glycogen as well as a large amount of lipids in the placenta [15]. Glycogen and lipids are known to disrupt IEF-separation by blocking the IPG-strip resulting in no or very few spots on 2D-PAGE. Hence, the centrifugation step was crucial for the sample preparation. The centrifugation did not cause a selective loss of proteins, indicating that most of the placental proteins remained in the supernatant. The freezing of samples directly after solubilization (without the centrifugation step) resulted in insoluble aggregates. However, freezing samples after the centrifugation step did not change the proteome map. This observation may facilitate the work flow in proteomics where many samples are analyzed, and the samples can be stored in the freezer until further use without having a major influence on the results.

Our optimization procedures showed that solubilizing with urea and CHAPS and precipitating with dichloromethane/methanol or acidified acetone gave the highest number of spots on a 2D-PAGE. Solubilization with Hepes buffer in combination with dichloromethane/methanol also gave a high number of spots (Figure 2, B1), although there was considerably more horizontal streaking. Dichloromethane/methanol has the advantage or also removing lipids, and since the placenta is rich in lipids, this precipitation method may be the preferred choice. Hence, in a first study urea/CHAPS in combination with dichloromethane/methanol was chosen to examine the differences between the PE and normotensive placental proteome.

The differentiating proteins have proven to be hard to identify due to the low concentration of the proteins in the differing spots. The same problem was highlighted in a recent preeclamptic proteomic study, where, as a consequence of low amount of proteins, none of the differentially expressed proteins could be identified [18]. However, we have so far been able to identify two proteins differing between PE and 


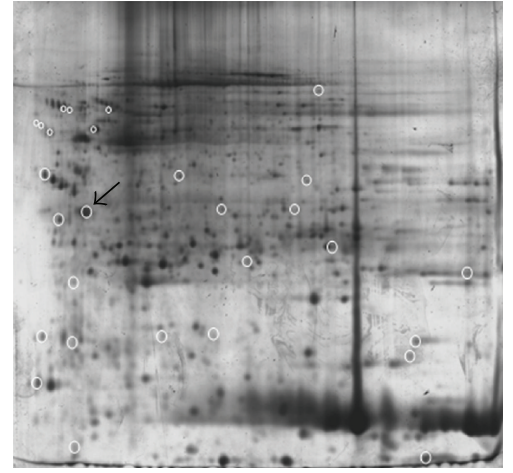

(a)

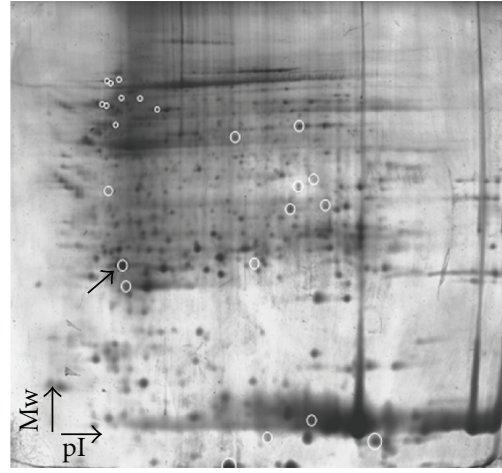

(b)

FIgURE 4: Two-dimensional separation of pooled proteins from normotensive (a) and PE (b). Circles indicate spots whose amount was increased in the 2D-PAGE of normotensive and PE samples analyzed with the Ludesi's software Redfin. Arrows show identified proteins; see Table 2. The gels were stained with SYPRO Ruby.

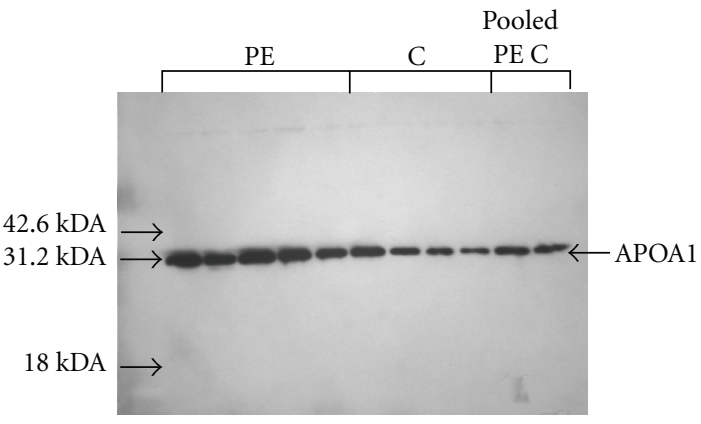

(a)

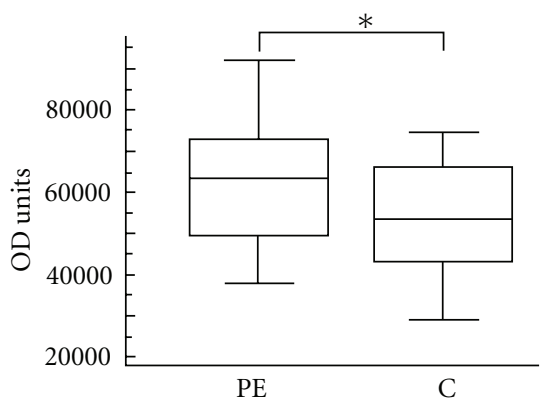

(b)

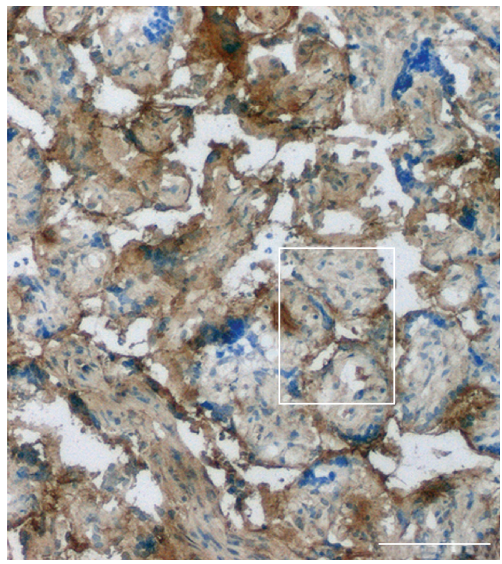

(c)

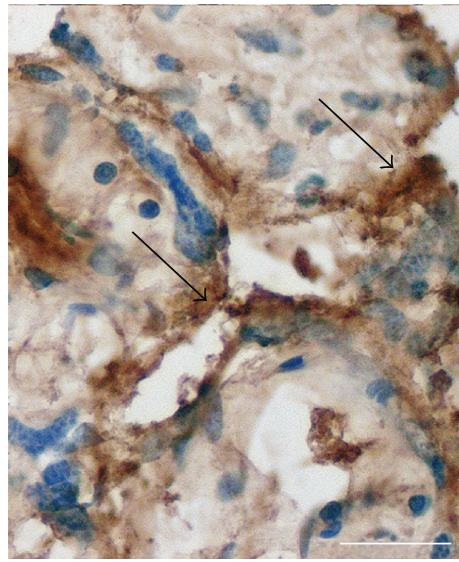

(d)

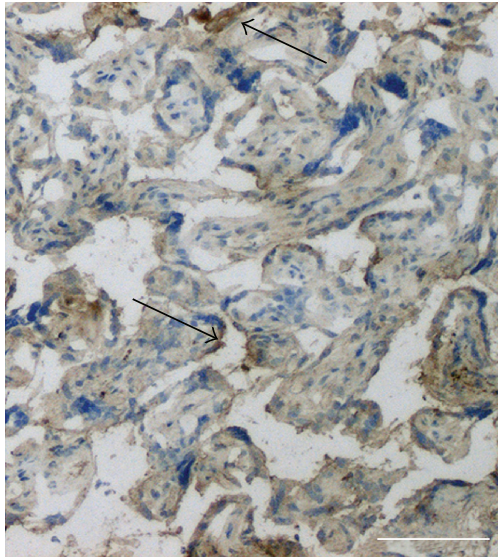

(e)

FIgURE 5: Validation of APOA1 accumulation. (a) A representative scanned image of Western blots for APOA1 (PE: $n=5$ and C: $n=4)$. All individual sample extracts were run (PE: $n=30$ and C: $n=30$ ). Expected size for APOA1 is $28 \mathrm{kDA}$. Molecular weight markers are represented by arrows. (b) Box plot for the intensity data for APOA1 as determined by GeneTools, showing the median and the 25th and 75th percentile. APOA1 was significantly increased in PE $(P=.02)$ (PE: 62247 (56597-67897) OD units, C: 53551 (48354-58749) OD units. Both values are presented as mean (95th percentile confidence interval). (c) Representative image for immunohistochemistry on APOA1 on a PE section. Scale bar $=100 \mu \mathrm{m}$. (d) Zoomed in image of APOA1 staining in a PE section (from the box in D). APOA1 staining was detected in trophoblasts (arrow). Scale bar $=30 \mu \mathrm{m}$. (e) A representative image of a control section. APOAl staining was weaker than in PE but was localized in trophoblasts as seen in PE. Scale bar $=100 \mu \mathrm{m}$. 
controls: APOA1 (increased in PE) and TPM3 (decreased in PE Figure 4). The increase of APOA1 in PE was validated by Western blot and immunohistochemistry (Figure 5). Due to the difficulties in identifying low abundance proteins in a crude extracted sample, one might have to do purifications of specific cell types, for example, trophoblast to explore the proteome of the placenta [24]. Another limitation with 2DPAGE is that membrane proteins do not easily enter IEF, the first dimension since they often precipitate in the IPG-strip.

In our first attempt to study differences between PE and normotensive placenta, we found apolipoprotein A1, a lipid binding protein, to be accumulated in the PE placenta. Of the lipoproteins, APOAl is mainly synthesized in the liver and intestines and is the major fraction of the high-density lipoproteins [25]. The placenta has also been suggested as a site of synthesis for APOA1 although it is not clear whether this is an actual de novo synthesis or an accumulation from the blood stream. In a recent whole genome microarray analysis, increased APOA1 gene expression was not detected in the PE placenta. It is unlikely that the accumulation of APOA1 in the PE placenta is due to high levels of APOA1 in the maternal plasma since plasma levels APOA1 are not increased in PE $[26,27]$. In a recent proteomics study of amniotic fluid, accumulation of pro-APOA1 was shown in the amniotic fluid in PE [28]. Hence, amniotic fluid may be a possible source of the accumulated APOA1 seen in the PE placenta. How the accumulation of APOAl affects the PE placenta is unknown. APOA1 may server either as nutrient or as a signaling molecule by affecting biological signaling pathways such as protein kinase C dependent pathway (PKC and the mitogen-activated protein kinases (MAPK) $[29,30]$.

In conclusion, two solubilization methods in combination with six different precipitation procedures were tested. Several steps in the sample preparation appear important. Removal of glycogen by centrifugation is crucial since the glycogen clogged the IPG-strip. Both the solubilization solution and the precipitation procedure affected the placenta proteome maps. In this study, solubilization with urea/CHAPS in combination with dichloromethane/methanol or acidified acetone proved to be the best precipitation procedures, resulting in more spots and less streaking on 2D-PAGE. Importantly, freezing samples following centrifugation of the solubilized samples did not affect the proteome map. However, due to the limitation using crude extracts for 2D-PAGE, low abundance proteins cannot readily be detected, and thus subcellular fractioning may be required to fully investigate the placenta proteome.

\section{Acknowledgments}

This work was supported by the Swedish Research Council: 5775, Anna Lisa and Sven Erik Lundgrens foundation for Medical Research, Craaford foundation, Magnus Bergvalls foundation, Swedish Society for Medical Research, Hospital of Lund Foundations, The Inga Britt and Arne Lundberg's Research Foundation, and the Royal Physiographical Society in Lund.

\section{References}

[1] H. N. Jones, T. L. Powell, and T. Jansson, "Regulation of placental nutrient transport-a review," Placenta, vol. 28, no. 8-9, pp. 763-774, 2007.

[2] V. Ganapathy, P. D. Prasad, and F. H. Leibach, "Use of human placenta in studies of monoamine transporters," Methods in Enzymology, vol. 296, pp. 278-290, 1998.

[3] J. S. Hunt, "Stranger in a strange land," Immunological Reviews, vol. 213, no. 1, pp. 36-47, 2006.

[4] J. C. Cross, "Placental function in development and disease," Reproduction, Fertility and Development, vol. 18, no. 2, pp. 7176, 2006.

[5] D. T. Krieger, "Placenta as a source of 'brain' and 'pituitary' hormones," Biology of Reproduction, vol. 26, no. 1, pp. 55-71, 1982.

[6] G. Blaich, B. Janssen, G. Roth, and J. Salfeld, "Overview: differentiating issues in the development of macromolecules compared with small molecules," in Handbook of Pharmaceutical Biotechnology, S. C. Gad, Ed., p. 77, John Wiley \& Sons, New York, NY, USA, 1st edition, 2007.

[7] S. A. Liebhaber, M. Urbanek, J. Ray, R. S. Tuan, and N. E. Cooke, "Characterization and histologic localization of human growth hormone-variant gene expression in the placenta," The Journal of Clinical Investigation, vol. 83, no. 6, pp. 1985-1991, 1989.

[8] J. W. Smith, D. J. Vestal, S. V. Irwin, T. A. Burke, and D. A. Cheresh, "Purification and functional characterization of integrin $\alpha(v) \beta 5$. An adhesion receptor for vitronectin," The Journal of Biological Chemistry, vol. 265, no. 19, pp. 1100811013, 1990.

[9] H. Konishi, S. Kuroda, Y. Inada, and Y. Fujisawa, "Novel subtype of human angiotensin II type 1 receptor: cDNA cloning and expression," Biochemical and Biophysical Research Communications, vol. 199, no. 2, pp. 467-474, 1994.

[10] J. M. Roberts and D. W. Cooper, "Pathogenesis and genetics of pre-eclampsia," The Lancet, vol. 357, no. 9249, pp. 53-56, 2001.

[11] I. A. Brosens, W. B. Robertson, and H. G. Dixon, "The role of the spiral arteries in the pathogenesis of preeclampsia," Obstetrics and Gynecology Annual, vol. 1, pp. 177-191, 1972.

[12] N. Soleymanlou, I. Jurisica, O. Nevo, et al., "Molecular evidence of placental hypoxia in preeclampsia," The Journal of Clinical Endocrinology \& Metabolism, vol. 90, no. 7, pp. 42994308, 2005.

[13] D. A. Hall, J. Ptacek, and M. Snyder, "Protein microarray technology," Mechanisms of Ageing and Development, vol. 128, no. 1, pp. 161-167, 2007.

[14] C. Wingren and C. A. Borrebaeck, "Antibody-based microarrays," Methods in Molecular Biology, vol. 509, pp. 57-84, 2009.

[15] N. M. Gude, C. T. Roberts, B. Kalionis, and R. G. King, "Growth and function of the normal human placenta," Thrombosis Research, vol. 114, no. 5-6, pp. 397-407, 2004.

[16] D. Wessel and U. I. Flugge, "A method for the quantitative recovery of protein in dilute solution in the presence of detergents and lipids," Analytical Biochemistry, vol. 138, no. 1, pp. 141-143, 1984.

[17] R. P. Webster and L. Myatt, "Elucidation of the molecular mechanisms of preeclampsia using proteomic technologies," Proteomics: Clinical Applications, vol. 1, no. 9, pp. 1147-1155, 2007.

[18] K. Mine, A. Katayama, T. Matsumura, et al., "Proteome analysis of human placentae: pre-eclampsia versus normal pregnancy," Placenta, vol. 28, no. 7, pp. 676-687, 2007. 
[19] F. Milne, C. Redman, J. Walker, et al., "The pre-eclampsia community guideline (PRECOG): how to screen for and detect onset of pre-eclampsia in the community," British Medical Journal, vol. 330, no. 7491, pp. 576-580, 2005.

[20] R. Hass and C. Sohn, "Increased oxidative stress in preeclamptic placenta is associated with altered proteasome activity and protein patterns," Placenta, vol. 24, no. 10, pp. 979-984, 2003.

[21] A. Shevchenko, M. Wilm, O. Vorm, and M. Mann, "Mass spectrometric sequencing of proteins from silver-stained polyacrylamide gels," Analytical Chemistry, vol. 68, no. 5, pp. 850-858, 1996.

[22] T. Berggård, S. Miron, P. Önnerfjord, et al., "Calbindin $D_{28 k}$ exhibits properties characteristic of a $\mathrm{Ca}^{2+}$ sensor," The Journal of Biological Chemistry, vol. 277, no. 19, pp. 16662-16672, 2002.

[23] S. Singh, D. W. Powell, M. J. Rane, et al., "Identification of the p16-Arc subunit of the Arp 2/3 complex as a substrate of MAPK-activated protein kinase 2 by proteomic analysis," The Journal of Biological Chemistry, vol. 278, no. 38, pp. 3641036417, 2003.

[24] J. M. Robinson, D. D. Vandré, and W. E. Ackerman IV, "Placental proteomics: a shortcut to biological insight," Placenta, vol. 30, supplement 1, pp. 83-89, 2009.

[25] A. L. Wu and H. G. Windmueller, "Relative contributions by liver and intestine to individual plasma apolipoproteins in the rat," The Journal of Biological Chemistry, vol. 254, no. 15, pp. 7316-7322, 1979.

[26] M. B. Cekmen, A. B. Erbagci, A. Balat, et al., "Plasma lipid and lipoprotein concentrations in pregnancy induced hypertension," Clinical Biochemistry, vol. 36, no. 7, pp. 575578, 2003.

[27] K. Winkler, B. Wetzka, M. M. Hoffmann, et al., "Triglyceriderich lipoproteins are associated with hypertension in preeclampsia," The Journal of Clinical Endocrinology \& Metabolism, vol. 88, no. 3, pp. 1162-1166, 2003.

[28] J. S. Park, K.-J. Oh, E. R. Norwitz, et al., "Identification of proteomic biomarkers of preeclampsia in amniotic fluid using SELDI-TOF mass spectrometry," Reproductive Sciences, vol. 15, no. 5, pp. 457-468, 2008.

[29] J.-M. Darbon, J.-F. Tournier, J.-P. Tauber, and F. Bayard, "Possible role of protein phosphorylation in the mitogenic effect of high density lipoproteins on cultured vascular endothelial cells," The Journal of Biological Chemistry, vol. 261, no. 17, pp. 8002-8008, 1986.

[30] Y. Q. Wu, E. V. Jorgensen, and S. Handwerger, "High density lipoproteins stimulate placental lactogen release and adenosine $3^{\prime}, 5^{\prime}$-monophosphate (cAMP) production in human trophoblast cells: evidence for cAMP as a second messenger in human placental lactogen release," Endocrinology, vol. 123, no. 4, pp. 1879-1884, 1988. 

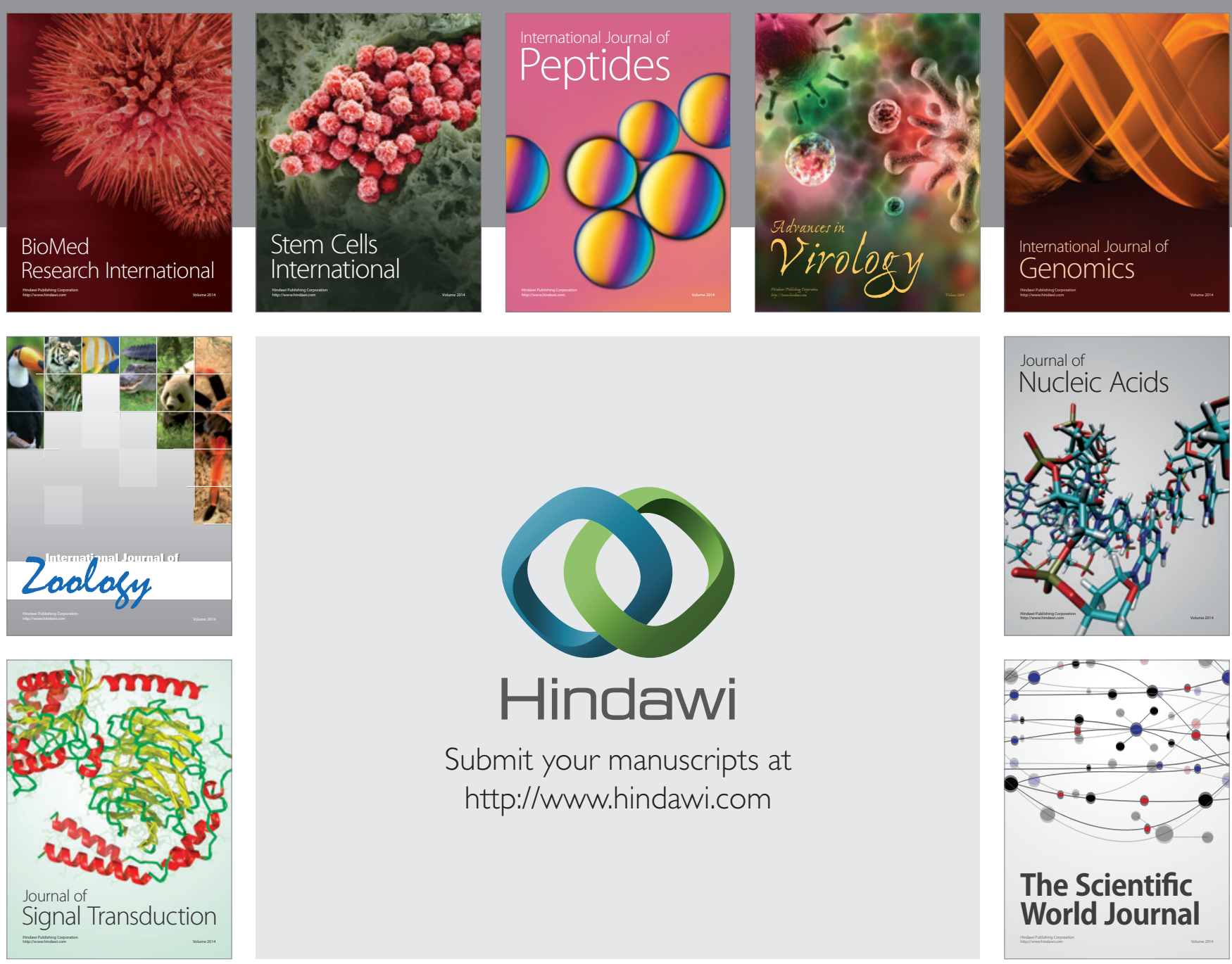

Submit your manuscripts at

http://www.hindawi.com
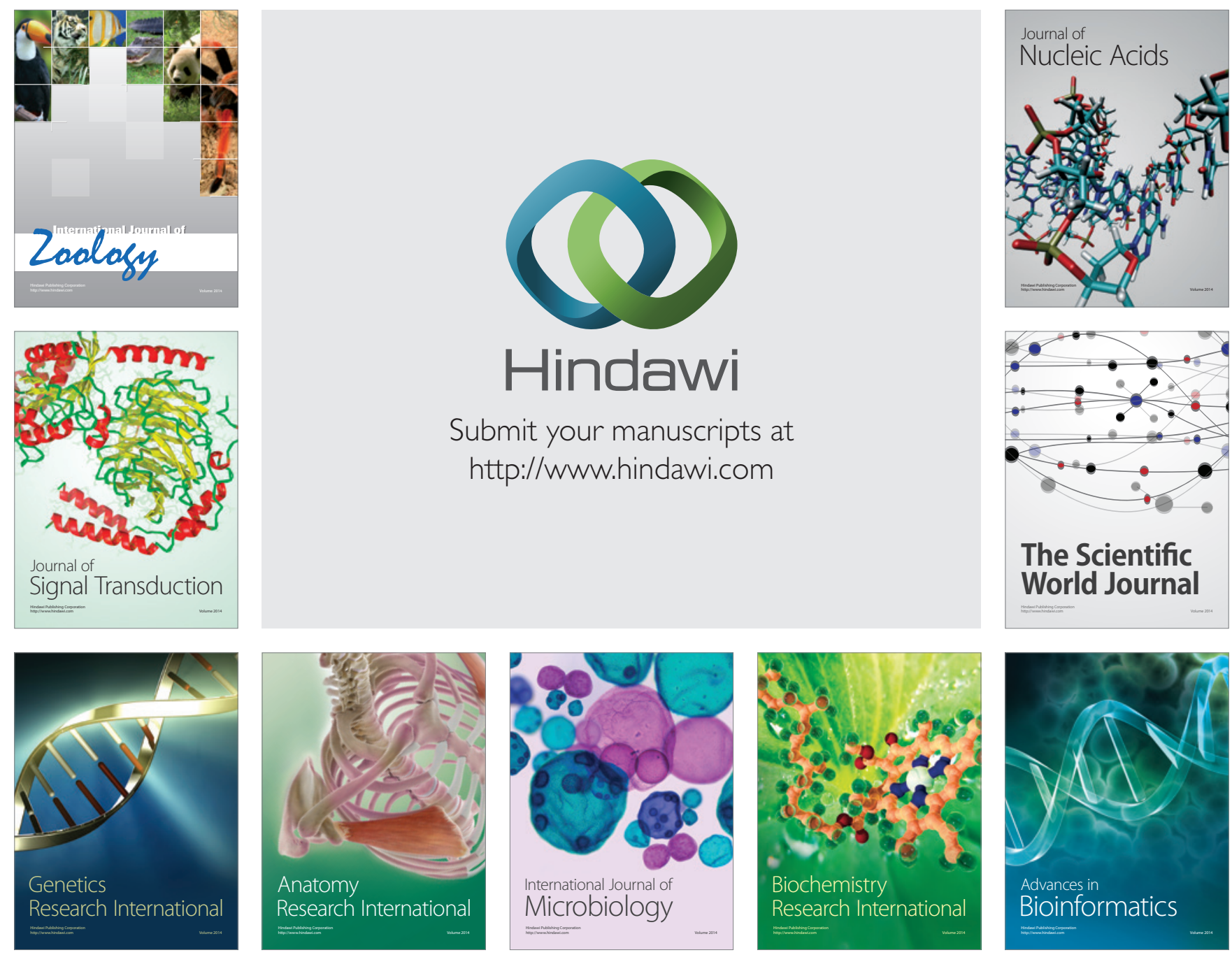

The Scientific World Journal
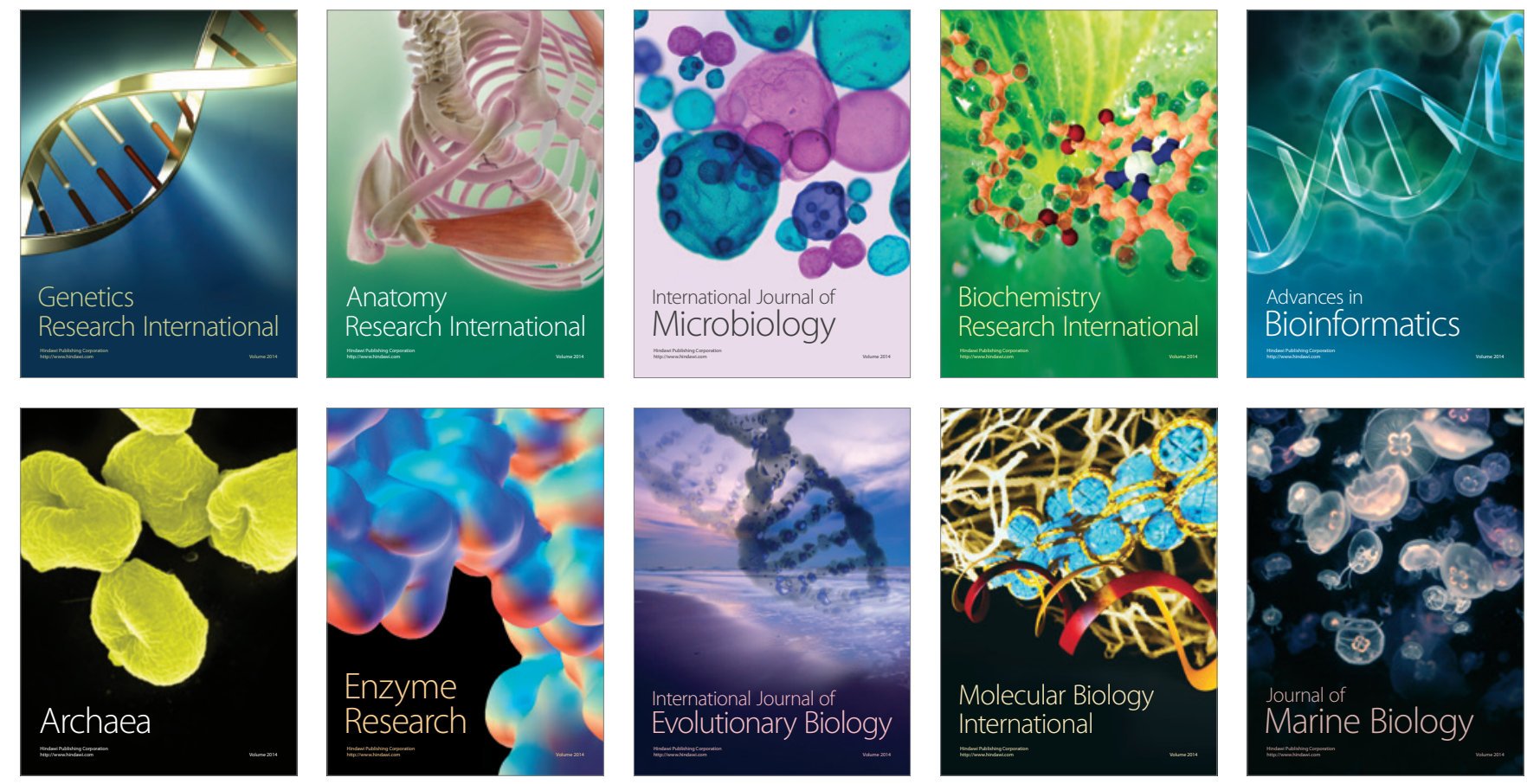